\title{
A Site- and Layer-Specific Sample Preparation Technique for Plan View TEM of Laser Diodes
}

\author{
J.Tanimura*, K.Kawasaki**, Y.Yoshida** and H.Kurokawa* \\ *Mitsubishi Electric Corporation, Advanced Technology R\&D Center, Tsukaguchi-Honmachi 8-1-1, \\ Amagasaki, Hyogo, Japan, 661-8661 \\ ** Mitsubishi Electric Corporation, High Frequency \& Optical Semiconductor Division, Mizuhara \\ 4-1, Itami, Hyogo, Japan, 664-8641
}

It is known that degradation phenomena of laser diodes often originate at specific site and/or on specific layers in the devices. In order to investigate structure of the degraded regions, cross sectional TEM observations have been carried out using a focused ion beam (FIB) technique.[1,2] However, plan view TEM observation is a powerful method for clarifying the degradation mechanisms. Petroff and Hartman pointed out that dark-line defects of GaAs-GaAlAs lasers are associated with dislocation networks generated from a threading dislocation.[3] Though they prepared plan view TEM samples using mechanical polishing and argon ion milling, this is not ideal method to thin samples at a specific site and at a specific layer. In this article, we have developed a site-specific and a layer-specific sample preparation technique for plan view TEM observations using FIB.

The sample preparation procedure is illustrated in FIG.1. Lasers, which are $300 \mu \mathrm{m}$ width $\mathrm{x} 900 \mu$ $\mathrm{m}$ length $\mathrm{x} 100 \mu \mathrm{m}$ thickness, were mechanically polished to the thickness of $50 \mu \mathrm{m}$ from the backside of the device. Shallow grooves along and perpendicular to the ridge near the degraded region were formed using FIB from the top surface of the device. Then, the area divided by grooves was cleaved off with a needle or a pincette under an optical microscope. At this step, the cross-section near the degraded region could become observable. After sample rotation by $90^{\circ}$, the quantum well layer was able to be thinned using a conventional FIB technique for preparing cross sectional TEM samples.[4] Accordingly, it enables us to observe the quantum well layer of the degraded region in the device from the direction normal to the device plane. A processing time of these preparation procedures was about 4 hours.

This technique was applied to investigate degradation mechanism of 980nm InGaAs/AlGaAs ridge waveguide lasers. The ridge width and the cavity length of the lasers are $3 \mu \mathrm{m}$ and $900 \mu \mathrm{m}$, respectively. $\mathrm{Au} / \mathrm{Ti}$ metals are evaporated on the ridge top and $\mathrm{Au}$ is plated on the metals leaving a distance of $25 \mu \mathrm{m}$ from both facets. The lasers investigated here were intentionally degraded under excessive current operation of about $1000 \mathrm{~mA}$. Under the operation condition, the degradation of the devices reproducibly occurs at the edge of the $\mathrm{Au}$ plating because of the local rise of temperature due to current concentration. Plan view TEM micrographs of degraded region are shown in FIG.2. FIG.2a. was taken at the front of the degraded region and FIG.2b. near the edge of the Au plating. Dislocations are on the (001) plane that is the quantum well layer and no threading dislocation was observed. Neither dislocations nor other defects were observed in lasers with the operating current below $900 \mathrm{~mA}$. Therefore, the degradation of the devices was not due to propagation of a threading dislocation reported by others.[5,6] The Burger's vector of the dislocations is found to be of the $(\mathrm{a} / 2)<011>$ type because the dislocation contrast is almost disappeared with a $<400>$ reflection. A dislocation loop at the front of the degraded region marked with a black arrow in FIG.2a was identified to be of the vacancy type using the inside-outside contrast method.(FIG.3.) During the device operation, vacancies were supersaturated and aggregated to form vacancy type dislocation loops. It is concluded that the propagation of the dislocation loops originated from the vacancy supersaturation causes the degradation of the lasers under the excessive current operation. 


\section{References}

[1] R.E. Mallard et al., Proc. SPIE Int. Soc. Opt. Eng, 3004, (1997) 145.

[2] Y. Yabuuchi et al., J.Electron Microscopy 48(1999) 791.

[3] P. Petroff et al., Appl.Phys.Lett. 23(1973) 469.

[4] J. Szot et al., J.Vac.Sci. \& Tachnol. B10(1993) 575.

[5] P. Petroff et al., J.Appl.Phys. 45(1974) 3899.

[6] P.W. Hutchinson et al., Appl.Phys.Lett. 26(1975) 250.
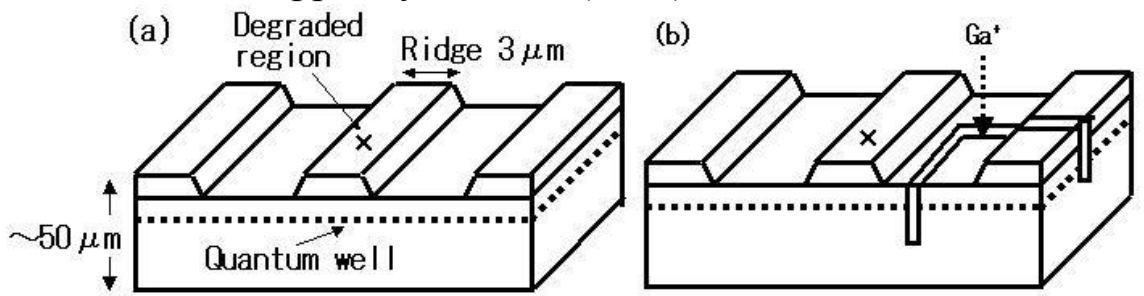

(c)

(d)
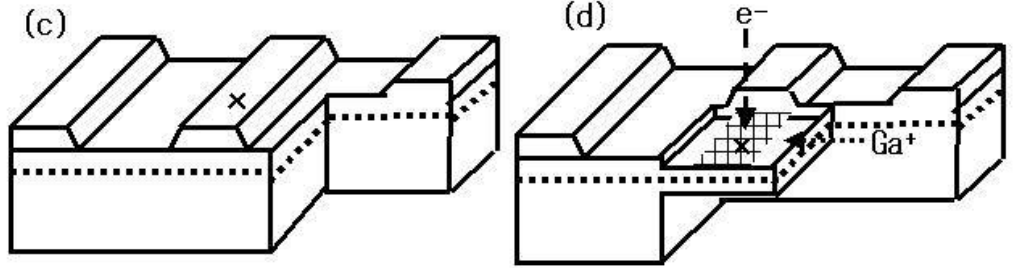

FIG.1. Schematic diagram of the sample preparation procedure.
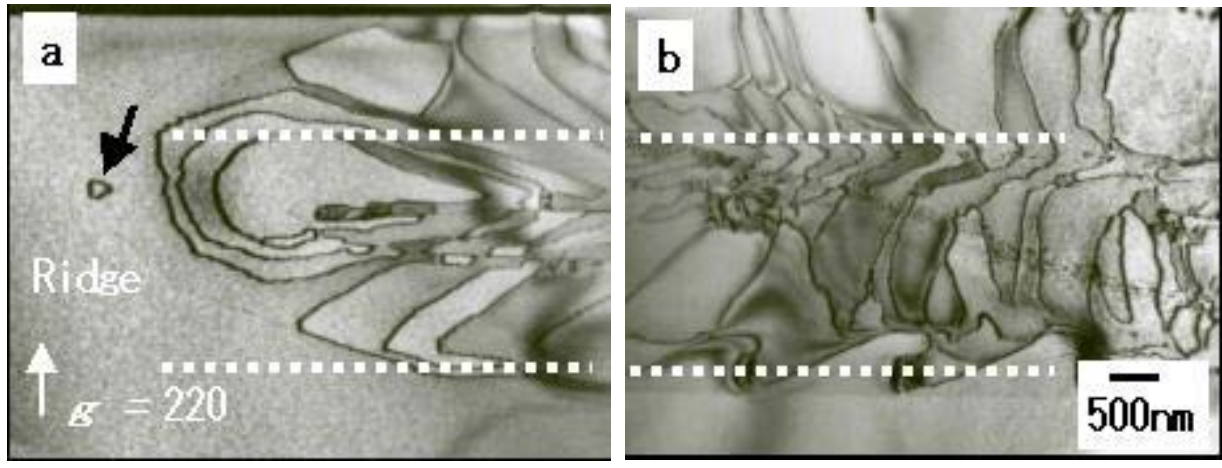

FIG.2. Plan view TEM micrographs of the degraded region. FIG.2a. was taken at the front of the degraded region and FIG.2b. near the edge of the Au plating.
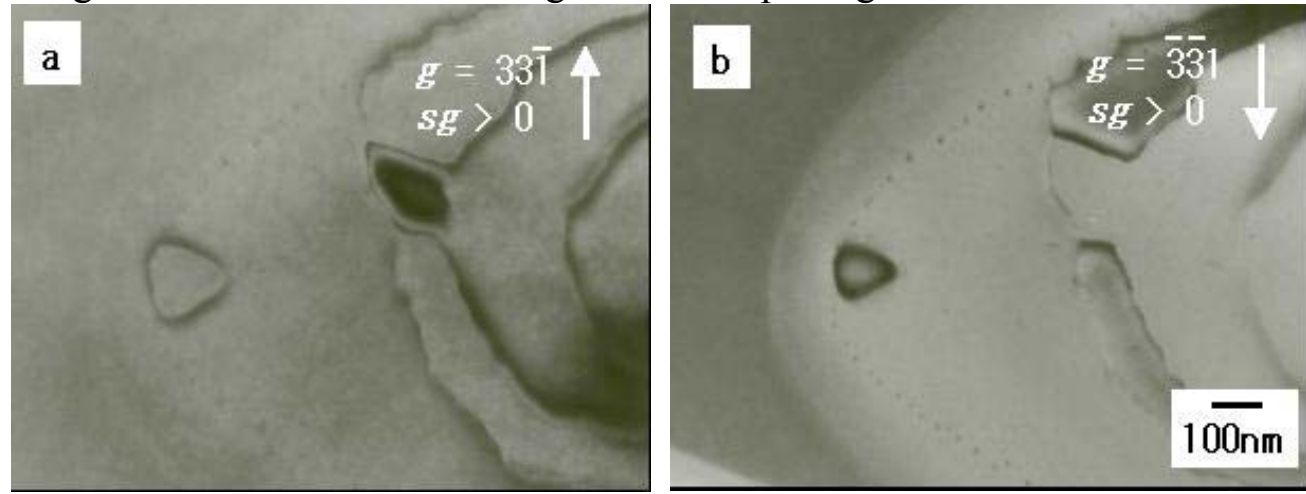

FIG.3. Bright-field TEM micrographs showing a dislocation loop at the front of the degraded region. The dislocation loop was observed as outside contrast in FIG.3a. taken with the reflection of $g=33-1$ and inside contrast in FIG.3b. with the reflection of $g=-3-31$. The deviation parameter $s g$ was positive in both cases. 